\title{
Hybrid Addressing Scheme for Preemptive Distributed Address Mechanism
}

\author{
Ketki P.Kardile-Gawali, Vinod V.Kimbahune \\ Department of Computer Engineering \\ STES's Smt. Kashibai Navale College of Engineering \\ Pune - 411041 India
}

\begin{abstract}
ZigBee is the most powerful standard for wireless sensor network.Pre-emptive Distributed Address Assignment (PDAA) Mechanism used to improves Zig-Bee address assignment and PDAA Mechanism Presents an auto-routing mechanism which doesn't store any information into routing table. There are two types of devices such as router and end device. But in ZigBee \& PDAA mechanism has a problem that there is parent $\&$ child relationship i.e. packet follow Tree topology to forward the packet from source to destination and second problem is address reuse mechanism.
\end{abstract}

So, This Paper Proposes a Hybrid routing scheme to reduce the routing cost by using the neighbor table that is originally defined in the ZigBee standard and Provide address reuse facility. In ZigBee and PDAA mechanism address reuse facility is a main issue so by using AODV routing protocol is used to provide address reuse facility.

\section{General Terms}

Distributed addressing

\section{Keywords}

Hybrid, routing, PDAA mechanism, ZigBee

\section{INTRODUCTION}

Recently, wireless sensor network consists of very high sensors for storing data, environmental control, and health monitoring. In [1] ZigBee is a standard protocol for address assignment by using hierarchical tree routing algorithm. Recently In wireless sensor network, ZigBee is used. ZigBee has many features Like Power-saving, low data rate and provide more flexibility and reliability. ZigBee uses a simple tree routing algorithm (HTR) to forward the packet from source to destination. In ZigBee, addresses are assigned using hierarchical tree routing algorithm that is each level calculate finite block addresses. It follows only parent-child relationship, so its routing cost is more. ZigBee doesn't maintain any routing information in the form of table.

In [2], used to develop the network quickly. Preemptive distributed address assignment mechanism means to preempt an address from parent neighbors.

Therefore, a preemptive mechanism is useful for ZigBee addressing mechanism. In PDAAM, auto-routing \& address Auto configuration mechanism are designed.

\begin{tabular}{|c|}
\hline Applications \\
\hline Application framework \\
\hline Network security \\
\hline MAC/physical layer \\
\hline
\end{tabular}

Fig 1 ZigBee Layer

This paper presents a Hybrid addressing scheme for Preemptive distributed address Mechanism by introducing neighbor tables, which is already the part of ZigBee specification. This paper proposes routers to check their neighbor tables before sending packets from source to destination or from parent to child. The actual novelty of the model lies in (a) Address assignment, (b) Address reuse.

\section{MOTIVATION}

In [2 \& 3] ZigBee \& PDAA mechanism, both are used for distributed address assignment. In [3] ZigBee follow a tree topology to forward the packets even if the destination is to close so its routing cost is more. Also ZigBee doesn't use the routing table to store the neighbor's information into routing table. In [2] PDAA maintain the routing information but doesn't provide address reuse facility.

\section{RELATED WORK}

ZigBee specification [3] presented a tree-based hierarchical routing algorithm (HTR). In a ZigBee network, the routers can directly transmit the packets from source to destination without maintaining any routing information. This routing mechanism is called hierarchical tree routing. Each parent is calculating it's a finite sub-block of the address space. This address space is used to allocate the address to its child.

Where,

Cm:-No of Childs,

$\mathrm{Rm}$ :-No of routers,

CSkip:-sub-block Address, Lm:-height of the network,

d: - depth of the network.

By given values $\mathrm{Cm}, \mathrm{Lm}$ and $\mathrm{Rm}$, we may calculate the CSkip (d) as follows:

CSkip $(\mathrm{d})=1+\mathrm{Cm}$. (Lm-d-1) if $\mathrm{Rm}=1$

\section{$\underline{1+\mathrm{Cm}-\mathrm{Rm}-\mathrm{Cm} \cdot \mathbf{R m}^{\mathrm{Lm}-\mathrm{d}-1}}$ 1-Rm otherwise}

Rules:

The address of the routers given by the following equation : 1 . Node $\mathrm{n}$ is router device. An = Aparent + CSkip $(d) .($ N-1) +1

2. Node $\mathrm{n}$ is end device. An = Aparent + CSkip (d).Rm+n 
In [2], PDAA mechanism introduces two steps. In the first step, nodes joined the network and addresses are assigned based on ZigBee distributed addresses assignment mechanism. New nodes directly getting addresses from their parents stop. In the second step, if address is not available then send Preempt request to parent neighbors.

Donator(node 21) consists of two data structures:Preempt_pending \& Preempted.Preemptor maintains a Preemption table.

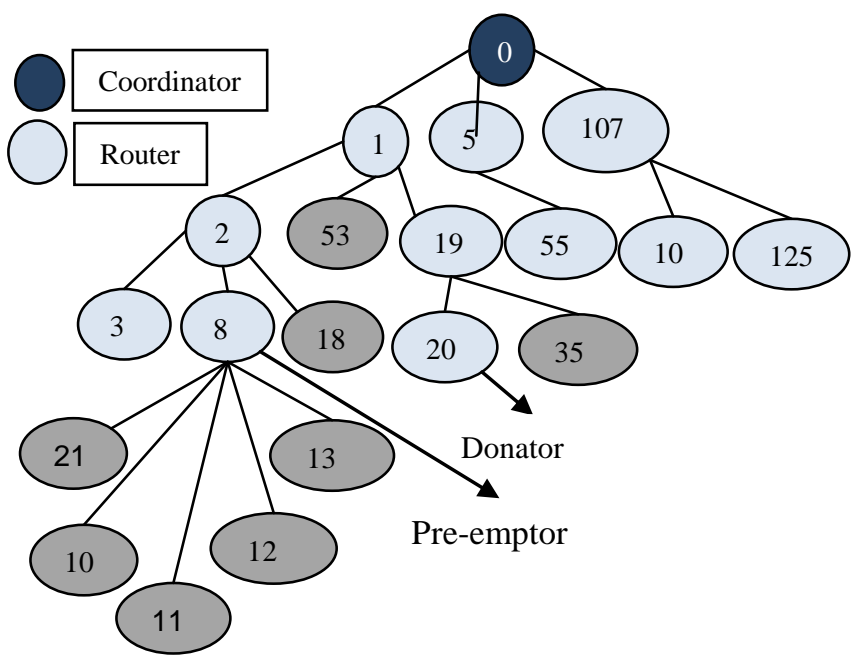

Fig 3.1 PDAA data structure

\subsection{Evaluation of related work}

Here previous work Table1 given below shows the evaluation of state of art.

Table 3.1 Evaluation

\begin{tabular}{|c|l|}
\hline $\begin{array}{c}\text { Previous } \\
\text { research } \\
\text { paper }\end{array}$ & \multicolumn{1}{|c|}{ Result/Conclusion } \\
\hline$[1]$ & $\begin{array}{l}\text { Used for Address Assignment using tree topology. } \\
\text { Problem: } \\
1 . \text { Packets follow the tree topology to forward the packets. } \\
\text { 2. Routing Cost is more. }\end{array}$ \\
\hline$[4]$ & $\begin{array}{l}\text {-reduce the routing cost by using neighbor table. } \\
\text {-Address Assignment using tree topology. } \\
\text { Problem: } \\
\text { 1. Doesn't reuse the address once it is assigned to Other } \\
\text { node. } \\
\text { 2.Does not record any routing information.(Neighbor table) } \\
\text { 3. Path duplication is occurring. }\end{array}$ \\
\hline$[2]$ & $\begin{array}{l}\text {-Distributed Address Assignment using tree topology } \\
\text {-Maintain Two Table: } \\
\text { 1.A preemptive address table } \\
\text { 2.Preempted Address } \\
\text { Problem: 1. Address reuse Problem when sensor died } \\
\text { without sending acknowledgment to its parent. }\end{array}$ \\
\hline
\end{tabular}

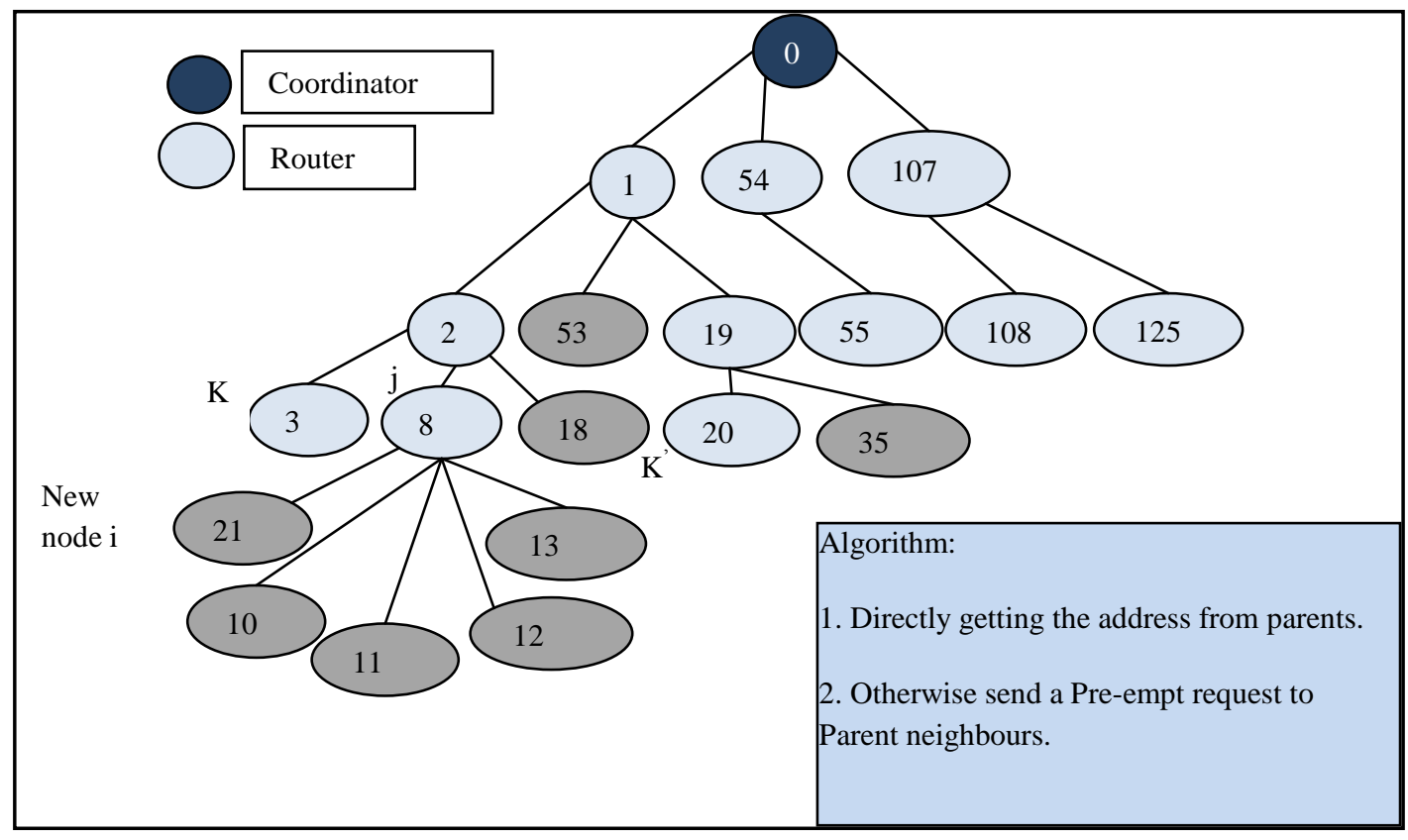

Fig 3.2 PDAA Preemption Mechanism 


\section{PROPOSED SYSTEM}

In this section, this paper proposes the tree-based Hybrid hierarchical routing algorithm that improves the existing PDAA hierarchical tree routing mechanism by using the neighbor table. The presented algorithm basically uses ZigBee routing algorithm. When preemption happened, a preemption table is used to guide the parent's node to forward the packets to the destination node.

In this section presents in detail how to preempt addresses from parent's neighbors and how node can reuse the address once node get fail. Here this paper presents a Hybrid model having no of routers to assign the addresses to each child node. Only the root node \& router can assign the addresses.

The detailed description of fig 4.1 is as follows.

1. User Takes an input from outside or to enter a input as a message i.e. set source and destination.
2. System accepts the inputs from user and starts some processing, forward this inputs to particular router.

3. Router checks this inputs i.e. source and destination into its routing table. If the destination address is not found then it will find the router and same process is repeated. Finally if the destination address is present into particular routing table, then that router send acknowledgment back to the user.

\subsection{Use case Diagrams for Address reuse:}

This use case diagrams shows how to reuse the address once The node gets fail. There are two possibilities:

1 . When node crashed \& 2 .when node wants to leave or join the network. This address reuse facility was not included in the previous system.

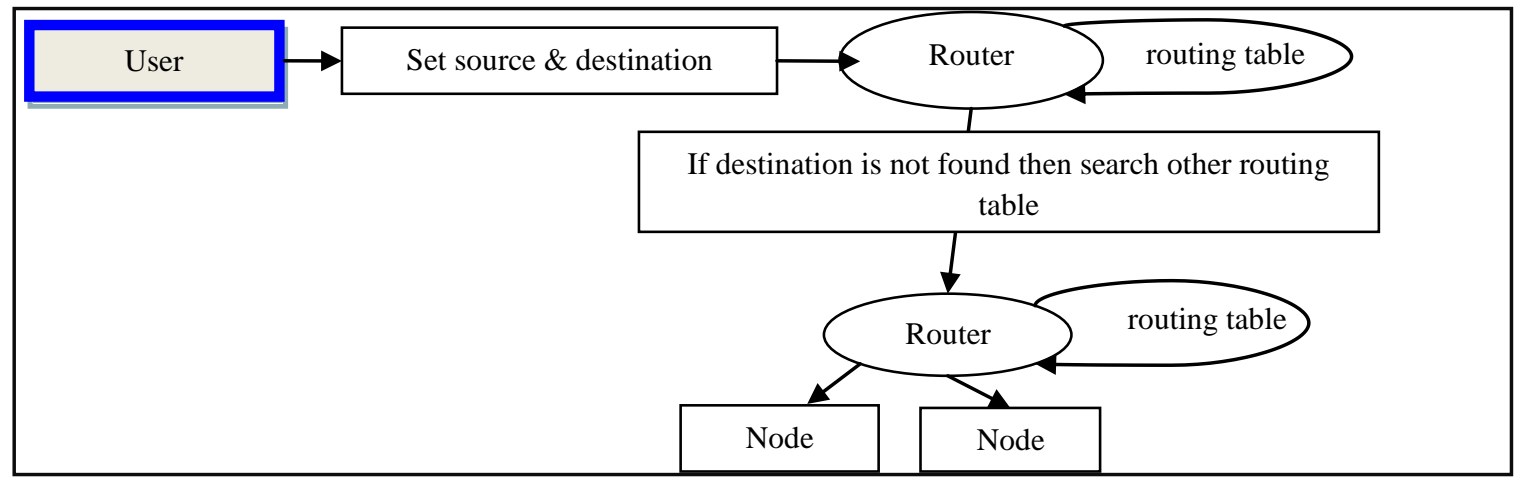

Fig 4.1 System Architecture

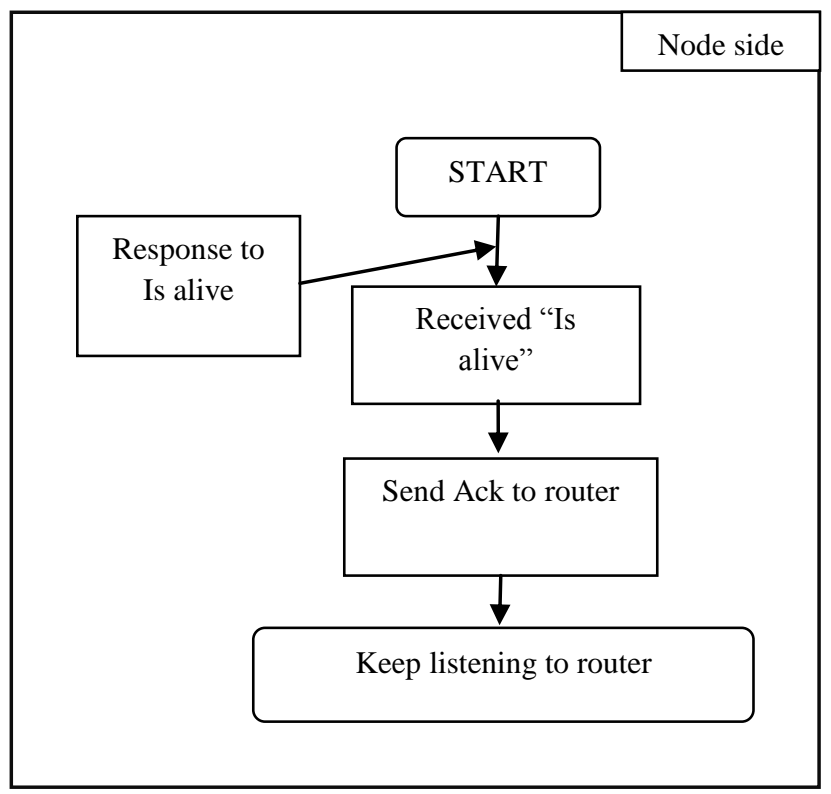

Fig 4.2 Address reuse node response

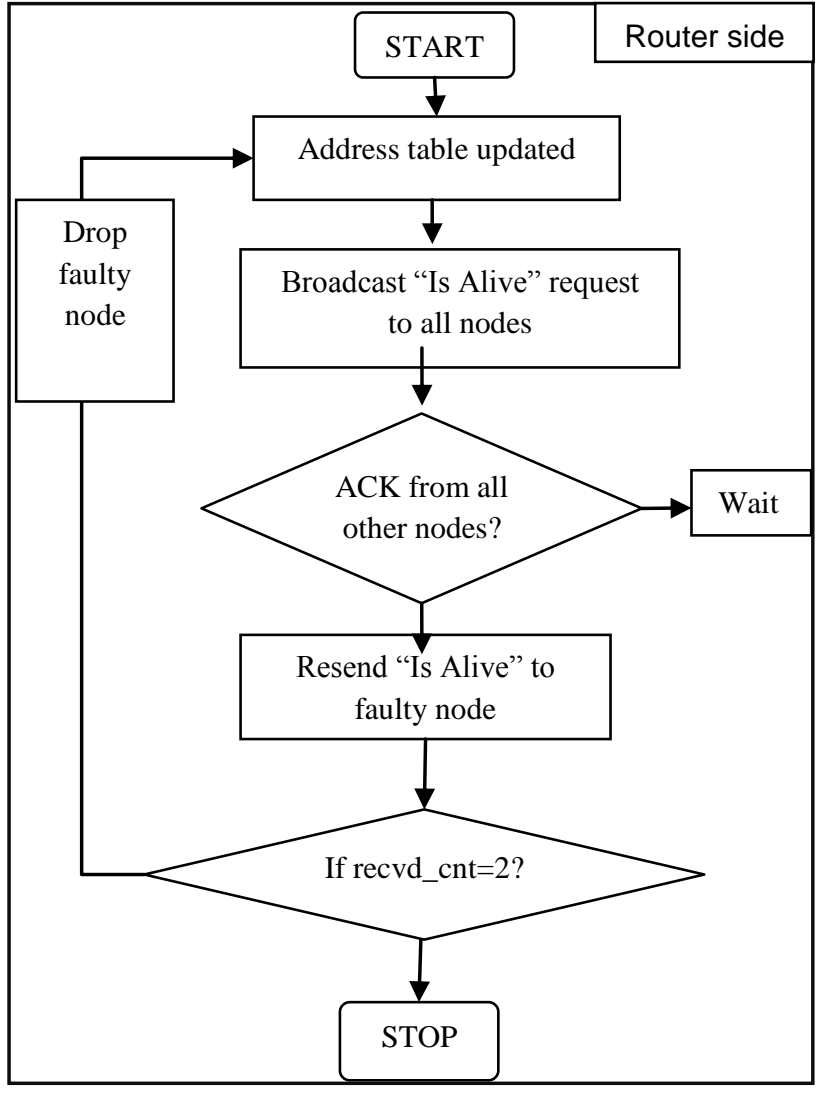

Fig 4.3 Address reuse router response 
This two diagram shows when node gets crashed, it immediately stops working. At the router side, router broadcast a "Is alive" message to all other nodes and waiting for reply. When node receive a message, each node send acknowledgement back to the router. Fail node doesn't send any reply. After router send same message to faulty node. When counter become 2, router free the address of faulty node. Second mechanism is to use hybrid algorithm to reduce the routing cost. In PDAA mechanism, system required more routing cost. In this system we are using Hybrid algorithm to reduce the routing cost and to avoid a reduplication of path.

\subsection{Proposed algorithm}

In this section presents algorithm for Hybrid address assignment. Input-source address (Sadr), destination address (Dadr) Output-depth (highest depth of common ancestor) Description of algorithm: -

From source address and destination address in routing packet, system can calculate all the common ancestors of the source node and the destination. Then the common ancestor with maximum depth is the minimum depth node in ZigBee hierarchical routing path. Initially $d$ is zero, $d$ represent the depth of the network. After that find out the common ancestor between source and destination by using step- 3 formula.

Algorithm:-

1. $d=0$

2. find out the common ancestor from source to destination.

$[($ Saddr - 1) $\bmod ($ Cskip $(d)]=[($ Dadr $)-1)] \bmod ($ Cskip $(d)]$

3. Do

Saddr $=[($ Sadr-1) $\bmod ($ Cskip(d) $] \operatorname{and}[($ Dadr $)-1)] \bmod ($ Cskip(d) $]$

4. $\mathrm{d}++$;

5. End while

6. Highest depth $=\mathrm{d}$

This algorithm helps for finding a common ancestor between source to destination and reduces the routing cost, to avoid path duplication.fig 4.2.1 \& fig 4.2.2 shows the results of these algorithm.

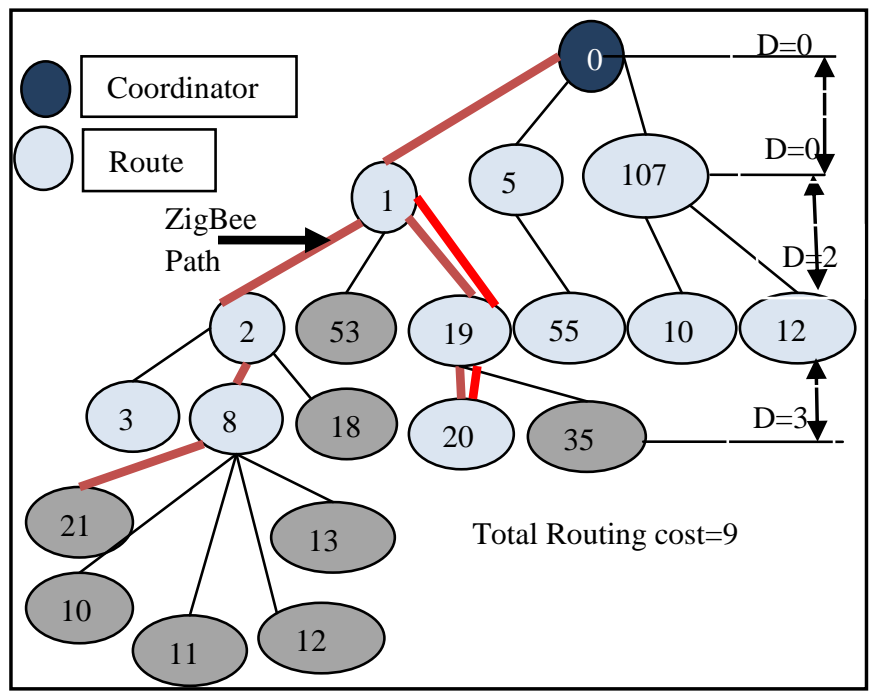

Fig 4.2.1 Existing mechanism

In this fig, consider 107 is source node \& destination is node 21.The resulting routing path is 107-0-1-19-20-19-1-2-821 (10 hops).by using hybrid algorithm the resulting routing path is 107-0-1-2-8-21(5 hops).As shown in fig 4.2.2, by finding common ancestor it reduces the routing cost. In these diagram 107 want to send a message to node 21 through common ancestor 1 .

\subsubsection{Use case Diagram for the address assignment}

Fig 4.2.3 shows the use case diagram for the Hybrid address assignment. Here user is able to create a message and set the source and destination. After that router will find out the entry's in the routing table and if entry is found then forward the result back to the user with current routing cost.

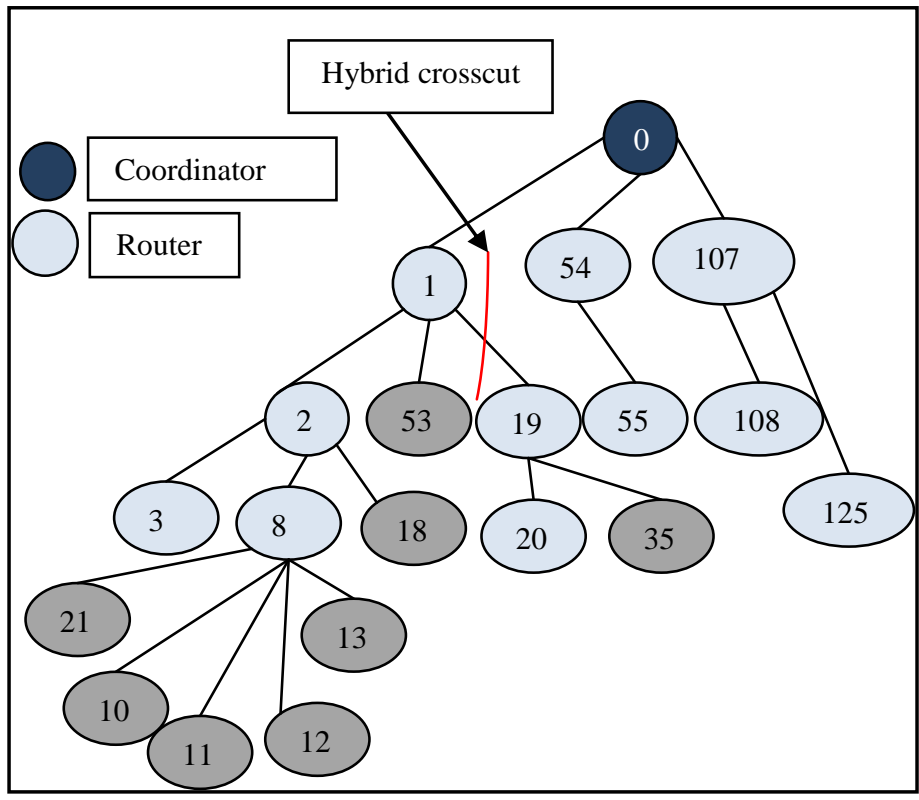

Fig 4.2.2 Hybrid concept

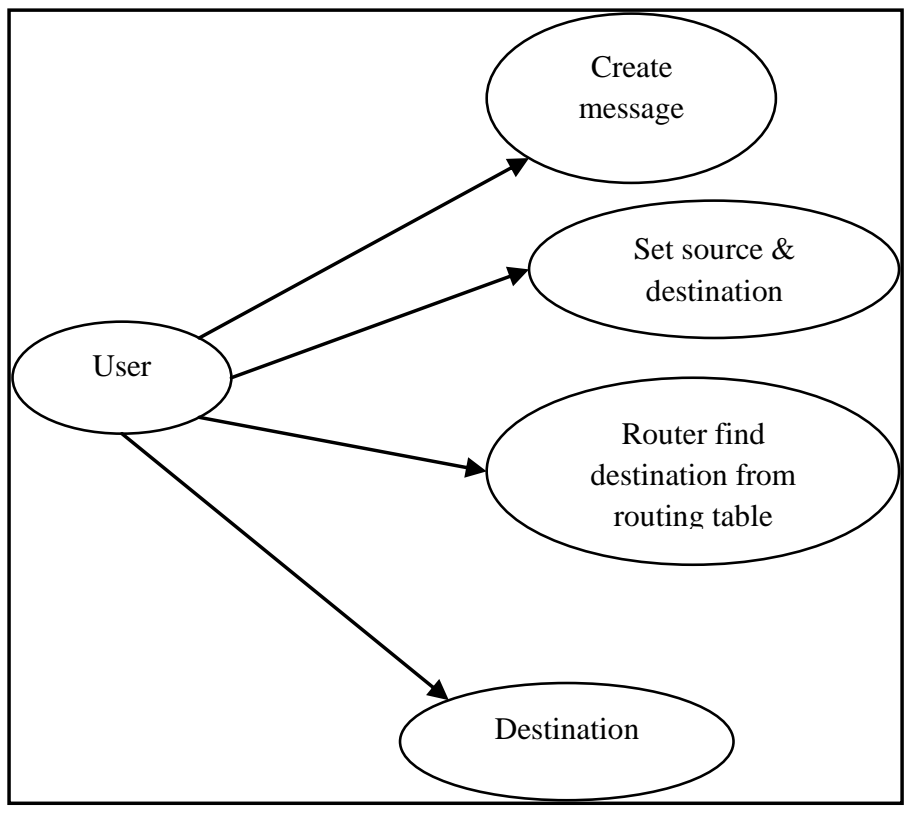

Fig.4.2.3 Use Case for Preemption mechanism

\subsubsection{Sequence Diagram}

Sequence diagram specifies the sequence of actions that are undertaken while operating the system.

1. Source sends a message with destination address.

2. Router checks its routing table. 
3. If destination is not found in the routing table then router will forward to another

Routers repeat above two steps.

4. If destination is found then inform the parent.

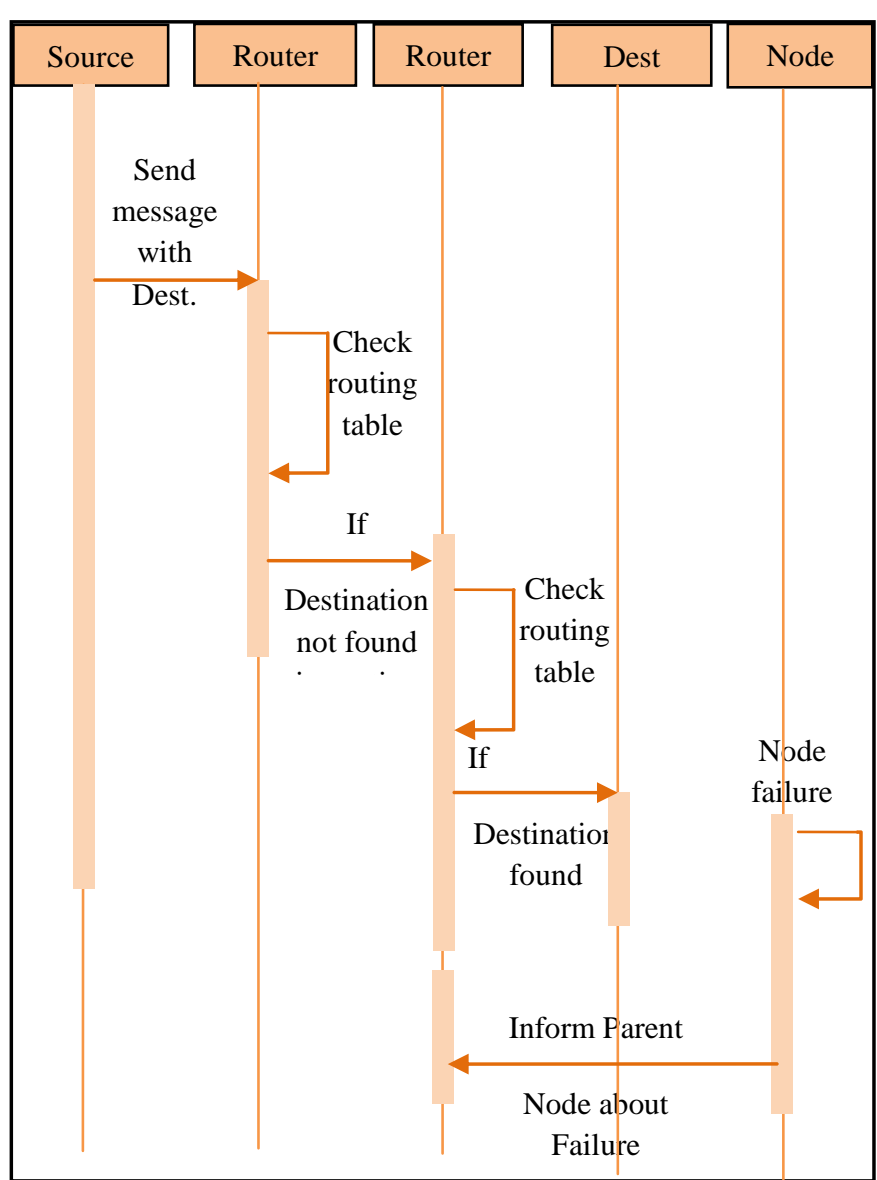

Fig 4.2.4 Sequence diagram

Fig 3.1 Donator maintain three tables and Preempted maintain 1 as follow:-

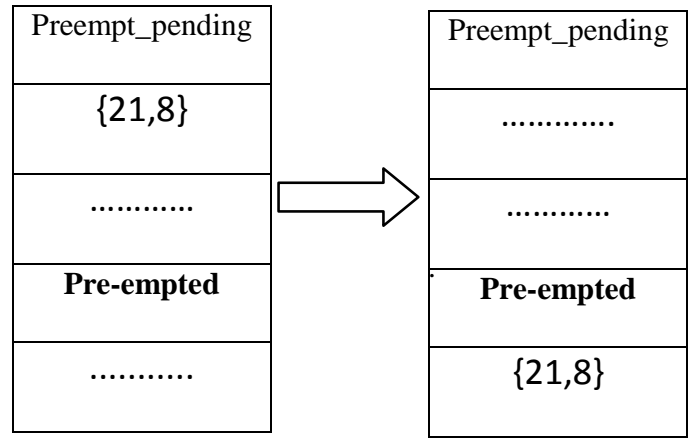

\begin{tabular}{|l|}
\hline Preemption_table \\
\hline$\{21\}$ \\
\hline
\end{tabular}

Table 4.2.4 three data structures

\section{CONCLUSIONS AND FUTURE WORK}

This paper proposes an address preemption mechanism and address reuse facility. Also address allocation scheme provides more flexibility because it provides a way to support Neighbors table. As the performance evaluation shows, the Hybrid tree routing reduces 30 percent of the routing cost needed for the PDAA tree routing algorithm.

Current and future work includes the implementation of the complete system to reduce the routing cost and to provide address reuse facility.

\section{REFERENCES}

[1] Z. Alliance, "http://www.zigbee.org," 2001.

[2] M. Fang, J. Wan, and X. Xu, "A Preemptive Distributed Address Assignment Mechanism for Wireless Sensor Networks," in The 4th IEEE International Conference on Wireless Communications, Networking and Mobile Computing, Dalian, China, 2008.

[3] Z. Alliance, "ZigBee specification," in ZigBee Document $053474 \mathrm{r} 13$

[4] K. Taehong, K. Daeyoung, P. Noseong, Y. Seong-eun, and T. S. Lopez, "Shortcut Tree Routing in ZigBee Networks," in Wireless Pervasive Computing, 2007.

[5] DMRep- K.Aberer, Z. Despotovic, "Managing Trust in peer to peer Information System", In Proc of the IX International Conference on Information and Knowledge Management, Atlanta, Georgia, 2001.

[6] Meng-Shiuan Pan,ZigBee-Based Long-Thin Wireless Sensor Networks: Address Assignment and Routing Scheme.

[7] Nitin H. Vaidya. "Weak Duplicate Address Detection in Mobile Ad Hoc Networks",ACM MobiHoc 2002, 206-216, (June 2002)

[8] R. Peng, S. Mao-heng, and Z. You-min, "ZigBee Routing Selection Strategy Based on Data Services and EnergyBalanced ZigBee Routing," in Services Computing,2006. APSCC '06. IEEE Asia-Pacific Conference on, 2006, pp. 400-404.

[9] "Wireless Medium Access Control (MAC) and Physical Layer (PHY) Specifications for Low-Rate Wireless Personal Area Networks (WPANs)," IEEE Std 802.15.42006ed: IEEE Computer Society, 8 September 2006.

[10] Akkaya K, Younis M. A survey on routing protocols for wireless sensor networks. The Elsevier Ad Hoc Network Journal, 2005,3(3):325-349.

[11] "Wireless Medium Access Control (MAC) and Physical Layer (PHY) Specifications for Low-Rate Wireless Personal Area Networks (WPANs)," IEEE STD 802.15.4 $4^{\mathrm{TM}}-2006$ ed: IEEE Computer Society, 8September 2006.

[12] M.Mohsin and R. Prakash. IP address assignment in a mobile ad hoc network. In Proc. MILCOM, pages 856861, 2002.

[13] An Overhead Reduction Strategy for Weak Duplicate Address Detection in Mobile Ad Hoc Networks, Hiraku Okada, Kazuki Akima, Kenichi Mase. 